\title{
Analysis and optimization of ultrasonic copper wires using fuzzy logic approach
}

\author{
Jacek Zawadzki ${ }^{1, *}$, Cezary Jędryczka ${ }^{2}$ \\ ${ }^{1}$ TKH Kabeltechniek, Engineering Department, 64-100 Leszno, Polska \\ ${ }^{2}$ Poznan University of Technology
}

\begin{abstract}
Ultrasonic welding has been used in the market over the past thirty years and offer to the manufacturing industries like automotive, aviation, medical and many more due to various hurdles faced by conventional fusion welding or crimp processes. Very short time (less than 1 second) and no additional material during the connection process are very key advantages. Due to this fact this technology can be used for mass production. Plenty of times, the problems faced by industry due to this process are the poor weld quality and strength of the joints surface. In presented study, the process parameters like vibration amplitude, weld pressure and weld time are considered in analysis of the welding of copper wires $(\mathrm{Cu}-$ ETP1). Experiment is conducted according to the fractional design with replications to get the responses like tensile shear stress and T-peel stress of the weld. Measured data are utilized next to develop fuzzy logic model between responses and predictors. As a quality is an very important issue in these manufacturing industries, the optimal combinations of studied process parameters are found using fuzzy logic approach.
\end{abstract}

\section{Ultrasonic welding technology}

Ultrasonic welding (USW) is a promising method for joining of softer type metals - in this case copper. The USW method has been developed between the 1940 and 1950s [1]. In ultrasonic welding method two metals surfaces are joined due to the friction like relative motion between them with a clamping pressure. During this motion the local surface roughness, contaminants and oxides present over it, deform and disappear and make metal to metal connection possible - see illustration of USW process shown in Fig. 1a) and 1b).

a) Ultrasonic equipment working scheme

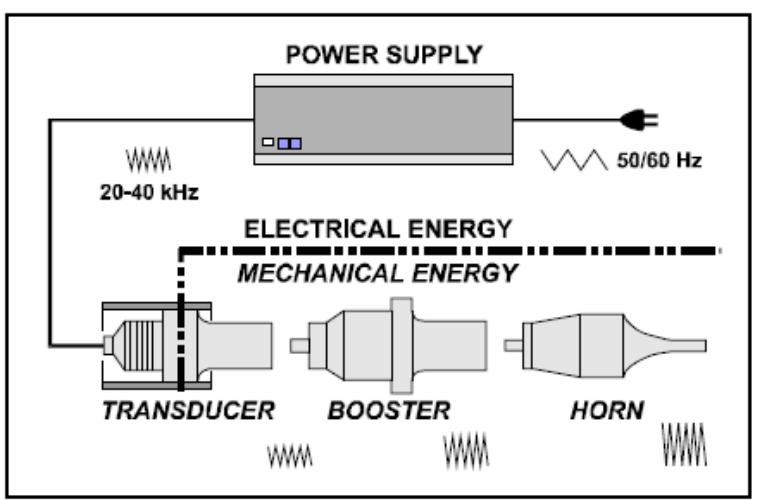

b) ultrasonic welding process

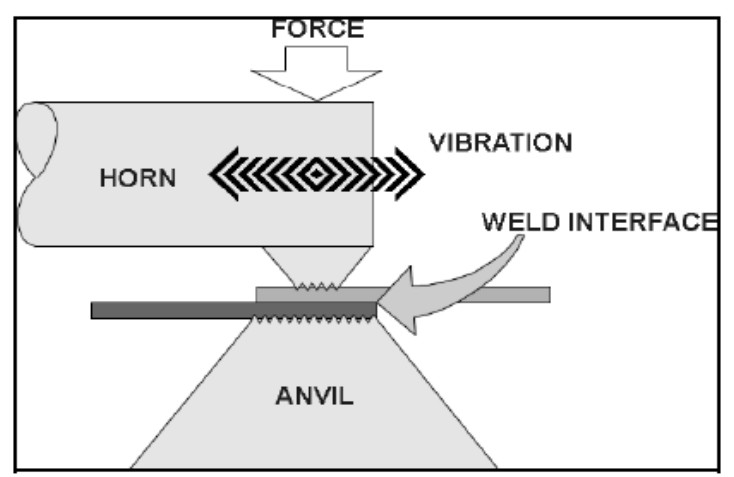

Fig 1. Ultrasonic welding process.

\subsection{Benefits of ultrasonic welding}

Ultrasonic metal welding exhibits unique welding properties that include:

- Excellent electrical, mechanical and thermal connections between similar or dissimilar metals;

- Low heat up during the ultrasonic process (no annealing of material);

- Compensation for normal surface variations of the material;

- Ability to clean surface oxides and contaminants prior to welding;

- Ability to weld large areas using minimal energy;

- Ability to weld thin materials to thin materials;

- Low cost per weld. 


\subsection{Experiment procedure}

During experiment the materials and tools listed below were used:

Material: copper wires type FLRYW-B $1.0 \mathrm{~mm}^{2}$ (manufacturer: Leoni).

Table 1. Copper Cu-ETP1 parameters.

\begin{tabular}{|c|c|c|c|}
\hline Material & Type & Composition & Density \\
\hline Copper & $\mathrm{Cu}-\mathrm{ETP} 1(\mathrm{E}-\mathrm{Cu})$ & $\mathrm{Cu} \geq 99,90 \%$ & $8,9 \mathrm{~g} / \mathrm{m}^{3}$ \\
\hline
\end{tabular}

Equipment:

- Ultrasonic welding machine: Branson 2032S;

- Pull and peel stress meas.: Macmesin MultiTest 2,5-d.

The ultrasonic welding involves a number of process parameters which can influence the welding performance characteristic. From numerus literatures studies and experimental trails, three most important parameters can be highlighted:

- Vibration amplitude: $21 \div 31 \mu \mathrm{m}$;

- Weld pressure: $15,6 \div 21,6$ Psi;

- Weld time: $0,52 \div 0,72 \mathrm{~s}$.

To check the weld quality measurement of pull force value $\left(\mathrm{F}_{\text {pull }}\right)$ and peel stress value $\left(\mathrm{F}_{\text {peel }}\right)$ have been performed using two ultrasonic welds as shown in Fig 2. For the pull and peel force tests the layouts of wires like shown in Fig. 2a and Fig. 2b were used, respectively.

a)

b)
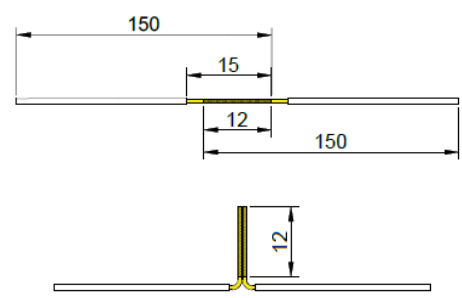

Fig 2. Layout of wires (all dimensions in millimeters).

To investigate the influence of all input variables on the responses, the fractional design DOEFE (Design of Experiment Factorial Design) was chosen. For preparing the input data set were used Minitab software. Structure of experiment was as follows: input factors -3 ; samples -12 ; blocks -3 .

\section{Fuzzy logic approach}

Fuzzy logic is a mathematical theory of inexact reasoning, which allows the modeling of the reasoning process of human in linguistic terms. The fuzzy logic control allows the existence of uncertain in handling parameters values [3]. Fuzzy logic system (Mamdani system) is mainly consisting of three important operations during its development i.e. fuzzifier, knowledge based inference engine and defuzzifier [4]. The fuzzifier uses membership functions to fuzzify the normalized values of each performance characteristic. Next, the inference engine (Mamdani fuzzy inference system) performs fuzzy reasoning on fuzzy rules to generate a fuzzy value. Finally the defuzzifer converts fuzzy predicted value into the output values $\left(\mathrm{F}_{\text {pill }}, \mathrm{F}_{\text {pull }}\right)$. To remove a certain degree of elusiveness the responses are normalized. Another reason behind the normalization process is that the membership function curve depend on the mapping on the input variables in its of $0-1$. In this computation, Higher-the-better (HB) criterion has been chosen for all quality characteristics. The formula used for $\mathrm{HB}$ criterion was:

$$
x_{i}(k)=\frac{y_{i}(k)-\min y_{i}(k)}{\max y_{i}(k)-\min y_{i}(k)}
$$

where $x_{i}(k)$ and $y_{i}(k)$ are the normalized data and observed data respectively the smallest and largest value of $y_{i}(k)$ are $\min y_{i}(k)$ and $\max y_{i}(k)$ respectively for $k t h$ responses.

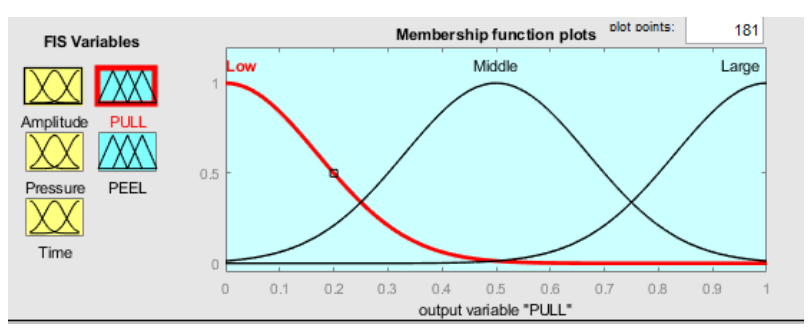

Fig 3. Membership function for individual responses (PULL).

Fuzzy value of quality characteristic is define by the membership function. In this analysis the input and output variables are expressed in terms of linguistic variables. The graphical representation of three fuzzy subsets i.e.: low, middle, large which is assigned to inputs (normalizes responses) have been illustrated in Fig. 3. The relationship between three inputs and two output were represent in the form if-then control rules. Mamdani implication is used to evaluate each rule and in this operation, the minimum membership of various fuzzy sets are take into account.

\section{Conclusions}

By using proposed fuzzy logic approach the multi parameter response of peel share stress force $\left(\mathrm{F}_{\text {peel }}\right)$ and pull stress force $\left(\mathrm{F}_{\text {pull }}\right)$ can be maximized using determined input parameters:

- Vibration amplitude: $28,5 \mu \mathrm{m}$;

- Weld pressure: 20,26 Psi;

- Weld time: $0,58 \mathrm{~s}$.

\section{References}

1. J.B. Jones, W.C. Elmore, C.F. De Frisco, , U.S. Patent, 2,946,119, (1960).

2. VersaGraphiX WSX Ergo Manual DCM00059 Rev First, American Technology.

3. C.C. Kao, A.J. Shih, S.F. Miller, J. Manuf. Sci. Eng. Trans. ASME 130 (6) (2008).

4. Y. Tzeng, F. Chen, Mater.Des. 28 (4) (2007), 11591168. 